\title{
Adquisición de habilidades metalingüísticas y enseñanza-aprendizaje de la composición escrita en educación primaria: reflexiones didácticas sobre los resultados de un estudio experimental
}

Eduardo Fernández de Haro

M. ${ }^{a}$ Pilar Núñez Delgado

Antonio Romero LóPez

Facultad de Ciencias de la Educación, Universidad de Granada

Recibido: 11 noviembre 2008 / Aceptado: 2 enero 2009

ISSN: 1697-7467

\begin{abstract}
RESUMEN: En este artículo se presenta un estudio experiemental llevado a cabo con alumnos de Educación Primaria con objeto de detectar su nivel de desarrollo de habilidades metalingüísticas y sus habilidades para la elaboración de composiciones escritas, así como las posibles influencias recíprocas entre unas y otras habilidades. A tal efecto se especifican los objetivos y el diseño de la investigación así como los resultados obtenidos y las correspondientes consideraciones didácticas.

Palabras clave: Didáctica de la lengua. Conciencia metalingüística. Enseñanza de la lengua escrita.
\end{abstract}

Metalinguistic Skills Acquisition And Teaching And Learning Of Written Composition In Primary Education: Didactic Reflections On An Experimental Study

ABSTRACT: This paper presents an experimental study carried out with Primary Education students in order to detect their metalinguistic skills developmental level along with their writing skills, and possible reciprocal influences. Thus, research aims and research methodology are specified, in addition to results and didactic considerations.

Key words: Language didactics. Metalinguistic awareness. Teaching of writing.

\section{ACtividad COGNitiva, Reflexión Metalingüística Y ENSEÑANZA de LA COMPOSICIÓN ESCRITA}

Puesto que el aprendizaje de la escritura requiere una enseñanza planificada y sistemática, es principalmente la escuela la institución responsable de que los escolares adquieran los niveles más elevados en el dominio de la lengua escrita, para lo que habría de promover las oportunas actividades reflexivas a través de su enseñanza-aprendizaje; sin embargo, no es esto lo que generalmente ocurre sino que, por el contrario, las más de las veces la escritura 
no llega a convertirse en verdadero instrumento de transformación del conocimiento, al no conseguir un aprendizaje capaz de sobrepasar los niveles ejecutivo y funcional y de alcanzar el nivel epistémico; y tal vez una de las principales razones de que esto ocurra así se deba a la escasa eficacia de las estrategias de intervención didáctica en la enseñanza de la escritura.

La metodología más tradicional se ha venido mostrando activa para el maestro y pasiva para el alumno: generalmente, el maestro impone el tema y se desentiende de la libertad del alumno, lee composiciones pero no ayuda a mejorar lo esencial de la composición escrita..., sin pensar que, en gran medida, las estrategias de enseñanza-aprendizaje, la actitud personal del profesor y el clima que se cree en la clase condicionan más la eficacia de esta enseñanza que las lecciones que pueden impartirse en relación con los temas de composición.

G. Rijlaarsdam y M. Couzijn ya comentaron en 1996 ([2000]: 215-221) que las deficiencias más importantes de los métodos más tradicionales son: 1.a) que la estimulación de la reflexión sobre el proceso de redacción es muy escasa; $2 .^{a}$ ) que, con frecuencia, los criterios acerca del buen o mal rendimiento no son claros; $3 .^{a}$ ) que las actividades de evaluación descansan en el docente y no en el alumnado; $4 .^{\mathrm{a}}$ ) que los alumnos casi nunca detectan o corrigen los fallos de su escritura; y $5 .^{\text {a)}) ~ q u e ~ e l ~ f e e d b a c k ~ s e ~ c o n c e n t r a ~ m a ́ s ~ e n ~ e l ~ p r o d u c t o ~ q u e ~}$ en el proceso. Estos autores concluyen afirmando, a modo de síntesis, que en la actualidad se puede decir que los puntos débiles del aprender haciendo se pueden resumir en la carencia de tres tipos de actividades que denominaron como: a) actividades de control (mediante la observación del propio comportamiento durante la tarea), b) actividades evaluativas (tanto de la calidad del producto obtenido como del proceso de escritura), y c) actividades reflexivas (a través de inferencias sobre la competencia de los propios alumnos como escritores y de sus saberes sobre la composición escrita), todas las cuales están libradas, a su juicio, a la iniciativa del estudiante en una pedagogía tradicional.

Sin embargo, además de superar las grandes deficiencias de los métodos tradicionales y el posible desconocimiento sobre los procesos cognitivos que intervienen en la producción de textos escritos, el profesorado ha de implicarse en la colaboración directa con los alumnos a lo largo del proceso de producción textual, escribiendo con ellos y ayudándoles a reflexionar al hilo de su proceso de aprendizaje hasta conseguir el dominio progresivo de esa técnica que es el escribir, y que, como tal técnica, es objeto de enseñanza-aprendizaje: de una enseñanza que colabore con los alumnos-escritores a elevar a nivel reflexivo lo que necesitan saber y lo que ya conocen o saben de forma intuitiva o práctica, de tal manera que se vaya produciendo un aprendizaje cada vez más autónomo, consciente y responsable de las técnicas necesarias para resolver los problemas que se presentan en cada una de las fases de desarrollo del complejo proceso de la producción textual, para cuya consecución no es suficiente, como pregonara la pedagogía tradicional, una didáctica de la composición escrita basada exclusivamente en la enseñanza de reglas y en el aprendizaje por medio de ejercicios. De aquí que para M. Castelló Badía (2000) muchas situaciones de enseñanza-aprendizaje no promuevan el proceso reflexivo de composición escrita característico de la función epistémica de la escritura, razón por la que, a su juicio, los estudios sobre tal enseñanza-aprendizaje todavía tengan un importante problema sin resolver: "explicar cómo es posible efectuar la transición desde una escritura escasamente reflexiva y lineal -consistente en volcar lo que se piensa sobre un papel- hacia un trabajo más reflexivo de composición, en el que el autor pueda controlar la producción de acuerdo a la interpretación y adaptación que haga de las características comunicativas" (p. 70). 
No basta, por tanto, solo el hacer, el redactar, sino que para conseguir un aprendizaje integrado y significativo de la composición escrita se hace imprescindible aprender componiendo, de tal manera que un buen alumno-escritor es aquel que escribe y aprende escribiendo, redacta y aprende redactando, produce textos y controla la producción, compone y aprende cómo componer. Es, por consiguiente, responsabilidad de la escuela promover tareas de reflexión a través de la enseñanza de esas estrategias didácticas que convierten el dominio progresivo de la expresión escrita en un instrumento de transformación del conocimiento y de desarrollo del pensamiento a través de un proceso reflexivo que atiende no solo a sus contenidos lingüísticos sino también a los cognitivos y pragmáticos.

A sabiendas de que no es tarea fácil encontrar una descripción teórica de carácter sistemático que se ajuste sin fisuras a la totalidad de los fines que se persiguen con la enseñanza de la lengua, en general, y de la composición escrita, en particular, desde el punto de vista didáctico, se viene insistiendo en la necesidad de encontrar un modelo bien fundamentado en lo lingüístico y en lo cognitivo que pudiera ofrecer una solución definitiva. Y tanto si nos referimos a los componentes lingüísticos como a los distintos subprocesos de elaboración textual subyacentes en cualquier modelo didáctico, se trata en todo caso de elevar a nivel reflexivo lo que en muchas ocasiones se conoce o se hace de manera intuitiva y práctica. Es este conocimiento reflexivo, llevado a cabo por los alumnos que aprenden a escribir, lo que les va a permitir ir adquiriendo progresivamente un mayor y mejor conocimiento sobre los saberes del lenguaje y sobre los procesos cognitivos de las tareas de escritura. Tal conocimiento reflexivo del escribir abarca no solo los elementos lingüísticos, procesuales y cognitivos sino también los conocimientos metacognitivos y las experiencias metacognitivas o sensaciones experimentadas conscientemente por el alumno-escritor en el acto de escribir.

No se olvide que la obra de Vygotsky (1934) presta especial atención a las relaciones entre lengua, desarrollo y aprendizaje de la lengua escrita, y resalta con extraordinaria importancia la función de la lengua escrita dentro del papel mediador del lenguaje en los procesos de aprendizaje por cuanto la lengua escrita, a diferencia de la lengua oral, se adquiere a edad más tardía y mediante un esfuerzo consciente que es necesario para el aprendizaje y desarrollo de su particular estructura y gramática específica, su diferente funcionamiento y su alto grado de abstracción; hasta el extremo de poder afirmar, como lo hizo su discípulo Luria (1979: 189), que "todo esto hace del lenguaje escrito un poderoso instrumento para precisar y elaborar el proceso de pensamiento".

De aquí que, al hilo de estas afirmaciones, Rijlaarsdam y Couzijn (2000: 221) hayan distinguido tres clases de enseñanza de la escritura según el nivel de atención que se preste al funcionamiento metacognitivo del alumno-escritor: (1) la enseñanza tradicional (en la que se ejercitan mediante la práctica varias subcapacidades concentradas en las actividades cognitivas de generación, estructuración, formulación, codificación y edición), (2) la enseñanza orientada al proceso (en la que la actividad docente se centra en enseñar a los alumnos-escritores a resolver problemas y regular su proceso de solución, estimulando las actividades cognitivas de formulación de propósitos, control de las actividades, revisión y evaluación del texto y revisión del enfoque de escritura), y (3) la enseñanza orientada al aprendizaje (que considera a los alumnos como aprendices autónomos, centrándose en actividades de formulación del propósito de aprendizaje, control de las actividades de aprendizaje, revisión/evaluación de las actividades y revisión del enfoque de escritura). Asimismo, diferencian cuatro paradigmas para la enseñanza de la escritura: (1) paradigma de solo tareas de ejecución (en el que el rol 
del escritor se centra en la escritura de un borrador, en la corrección del mismo y en la producción del texto definitivo), (2) de auto-reflexión (que supone el paso de la simple ejecución a la autorreflexión y la reflexión sobre la propia escritura), (3) de escritura comunicativa activa (en la que cobra especial relevancia la contemplación del rol de lector y la experiencia de los efectos textuales sobre el mismo) y (4) de escritura comunicativa reflexiva (paradigma más reflexivo en el que se potencian la capacidad para apreciar la calidad de los textos y el rol de comentarista del alumno-escritor).

Parece, pues, razonable pensar que no haya que conformarse con una enseñanza basada en el aprendizaje por tareas para conseguir el mejor grado de dominio de la composición escrita, ni tampoco parece que para ello baste con la sola reflexión sobre el proceso de composición, sino que hace falta la reflexión metalingüística; y no basta, como ocurre en el enfoque centrado en el proceso de escritura, con que la orientación de esta reflexión se focalice solo en las exigencias del contexto retórico (intención, objetivos, destinatario) obviando la atención requerida por el procesamiento lingüístico: el aprendizaje de la composición escrita necesita de la ejecución, de la reflexión sobre el proceso y de la reflexión metalingüística en relación con el contexto de producción textual.

En esta línea de consideraciones pedagógicas, es sobre todo a partir del último tercio del pasado siglo cuando van apareciendo distintas publicaciones que entienden los conocimientos metalingüísticos como un conjunto de habilidades que implican un saber reflexivo sobre el lenguaje por parte de sus usuarios que, al tiempo que opera en la actividad comunicativa, se enriquece con el ejercicio de esta actividad, ya se trate de lengua oral o de lengua escrita.

Resultan de especial interés para el tema que nos ocupa los trabajos sobre actividad metalingüística y enseñanza-aprendizaje de la escritura realizados en las universidades de Ginebra por los profesores J. Dolz, S. Erard y L. Allal, en la de Ámsterdam por G. Rijlaarsdan y M. Couzjin y en la Autónoma de Barcelona por A. Camps, M. Milian, O. Guasch y T. Ribas. A lo que hay que sumar las extraordinarias posibilidades que ofrecen los trabajos de M. ${ }^{a}$ A. Pinto, R. Titone y M. ${ }^{a}$ D. González Gil (2000) sobre medición de habilidades lingüísticas y metalingüísticas en escolares de distintos niveles del sistema educativo, gracias a las cuales es posible abrir un interesante espacio de investigación en el ámbito de la educación primaria sobre las posibles relaciones entre habilidades metalingüísticas y enseñanza-aprendizaje de la composición escrita.

Los resultados de esta investigación podrían ofrecer orientaciones más precisas para afrontar con mayores garantías de éxito una enseñanza tan compleja como es la de la composición escrita, y poder contribuir de esta manera a enriquecer la oferta de estrategias didácticas que pudieran ayudar a que los alumnos de educación primaria llegaran a adquirir el necesario desarrollo de unas habilidades metalingüísticas que les permitiera mejorar sus capacidades de producción de textual.

\section{OBJETIVOS DEL ESTUdiO Y DISEÑO DE LA INVESTIGACIÓN EXPERIMENTAL}

\subsection{Objetivos}

Con la intención de colaborar al esfuerzo realizado por tan importante número de estudiosos como los que acabamos de mencionar, que han orientado gran parte de su labor investigadora hacia el estudio de la actividad metalingüística y la enseñanza-aprendizaje de 
la escritura, se ha querido centrar esta investigación en el estudio de las habilidades lingüísticas y de las propiedades o componentes textuales de la composición escrita, con objeto de esclarecer las posibles relaciones entre adquisición y desarrollo de habilidades metalingüísticas, por una parte, y producción de textos escritos en alumnos de educación primaria, por otra; por lo que, teniendo en cuenta la situación actual en la que se encuentran tanto los trabajos sobre habilidades metalingüísticas como los referidos al aprendizaje de la composición escrita en educación primaria, este trabajo se ha orientado hacia la consecución de los siguientes objetivos:

1) Describir, por una parte, el grado de dominio que poseen los escolares de educación primaria sobre las propiedades o componentes textuales en la composición de textos escritos, y, por otra parte, el nivel de desarrollo de sus habilidades metalingüísticas.

2) Estudiar las relaciones existentes entre el grado de dominio de los diferentes componentes textuales que caracterizan el texto escrito y el nivel de desarrollo de las habilidades metalingüísticas de los alumnos.

3) Ofrecer algunas consideraciones de interés didáctico basadas en las relaciones existentes entre habilidades metalingüísticas y enseñanza-aprendizaje de la composición escrita.

\subsection{Diseño de la investigación}

Con el fin de conocer el grado de dominio para la elaboración de textos escritos y el de las habilidades metalingüísticas de los sujetos participantes en la investigación así como la relación existente entre ambos grados de dominio, se ha optado por llevar a cabo una investigación de tipo descriptivo. Asimismo, de los diferentes diseños de investigación existentes -transversal, longitudinal y secuencial- se ha escogido el transversal por entender que es el que mejor se adapta a la forma en que se ha determinado llevar a cabo este proceso de investigación, que se ha iniciado con la recogida de la información de toda la muestra en un solo momento, para elaborar y analizar con posterioridad los datos obtenidos.

Dadas las características de este tipo de investigación, no debe escapar a nuestra consideración que no se trata de llegar a determinar la causalidad exacta del fenómeno que se está estudiando, aunque sí es posible aproximarse a las causas que lo definen y clarifican, puesto que los datos que se obtienen a partir de la realización de las pruebas por los participantes brindan información acerca del grado de dominio que éstos poseen tanto sobre la composición de textos escritos como sobre el nivel de desarrollo de las habilidades metalingüísticas, además de que los estudios estadísticos realizados sobre los datos obtenidos proporcionan información acerca de las posibles relaciones entre estos aspectos.

\subsection{Participantes}

La muestra sobre la que se ha trabajado la componen escolares de ambos sexos (6 alumnos y 82 alumnas), pertenecientes a tres grupos de $6 .^{\circ}$ curso de educación primaria (1112 años de edad) de un colegio concertado de Granada capital, cuya docencia corre a cargo de la misma profesora de Lengua y Literatura, y cuya distribución es la siguiente: 
Tabla 1. Distribución de los participantes por grupos.

\begin{tabular}{|c|c|c|c|}
\hline GRUPOS & N & \% & \% AC. \\
\hline A & 29 & 32,9 & 32,9 \\
\hline B & 29 & 32,9 & 65,8 \\
\hline C & 30 & 34,1 & 100,0 \\
\hline TOTAL & 88 & 100,0 & \\
\hline
\end{tabular}

\subsection{Variables estudiadas}

Se especifican a continuación las variables estudiadas y los contenidos que las definen, para cada uno de los dos ámbitos investigados: composición escrita y habilidades metalingüisticas.

(1) En relación con la composición escrita:

a) Coherencia, referida al dominio del saber para expresarse de conformidad con los principios generales del pensar y con el conocimiento general de las cosas; al contenido de la información necesaria para expresarse sin excesos ni lagunas; al orden en la exposición de las ideas; y al dominio de la estructura del texto mediante la correcta disposición de sus párrafos.

b) Cohesión, que se centra en el dominio apropiado de campos léxicos; en el uso adecuado de las figuras de repetición (anáfora en sus distintas formas); en la utilización de deícticos espaciales y temporales; en el empleo de enlaces, conectores y marcadores textuales; y en la puntuación.

c) Corrección, que abarca la corrección morfosintáctica -construcción oracional-; la corrección léxica y precisión en el uso del vocabulario; y la corrección ortográfica: ortografía literal y acentuación.

d) Adecuación, que hace referencia al discurso adecuado al objeto sobre el que se escribe, apropiado respecto al destinatario y oportuno respecto a la situación; a la claridad de propósito(s) y objetivo(s) del texto; y al mantenimiento del nivel de formalidad en todo el discurso.

e) Presentación, relacionada con la legibilidad (caligrafía o dominio del trazado); la limpieza (ausencia de borrones, enmiendas, tachaduras...); y la correlación entre espacios en blanco y texto (formato, márgenes, título, espaciado interlineal).

f) Estilo, que se manifiesta en la variedad y riqueza léxica; en la complejidad y variación sintáctica; en la elegancia en la expresión; y en la creatividad expresiva.

g) Total de la prueba de composición escrita, referida al dominio global de los distintos componentes textuales específicos de la composición escrita.

(2) En relación con las habilidades metalingüísticas:

a) Comprensión metalingüística, entendida como capacidad para determinar los criterios que responden a la explicación de relaciones semántico-gramaticales en textos diferentes. 
b) Sinonimia metalingüística, referida a la capacidad para justificar satisfactoriamente las respuestas dadas en la captación del sentido ambivalente presente en textos diferenciados por su estructura sintáctica y/o por su significado.

c) Aceptabilidad metalingüistica, o capacidad para justificar juicios de aceptabilidad de textos con anomalías de carácter semántico.

d) Ambigüedad metalingüística, o habilidad para reestructurar el significado de un texto en función de los cambios de significado de un lexema.

e) Función gramatical metalingüística, entendida como capacidad para profundizar en los elementos del análisis de las funciones gramaticales (sujeto, objeto y predicado) y de las acciones y de los roles que se organizan en torno a ellas, así como de complementos circunstanciales y de oraciones subordinadas causales o finales.

f) Segmentación fonémica metalingüistica, o capacidad para captar el paso del plano del fonema al plano del morfema.

g) Puntuación total de habilidades metalingüísticas, que se extiende al dominio del conjunto de estas habilidades metalingüísticas globalmente consideradas.

\subsection{Instrumentos de medida}

Para la medida de las variables anteriormente expuestas se han utilizado dos instrumentos: (1) la Guía de evaluación de la composición escrita (GECE) -adaptación de la de J. Rodríguez Polo y A. Romero López (2004)-, cuyos componentes se recogen en la siguiente tabla, en la que también se hacen constar sus rangos parcial y total y sus coeficientes de ponderación:

Tabla 2. Componentes textuales de la composición escrita $y$ coeficientes de ponderación.

\begin{tabular}{|c|c|c|c|}
\hline VARIABLES & RANGO & COEF. POND. & RANGO TOTAL \\
\hline Coherencia & $1-5$ & 3 & $3-15$ \\
\hline Cohesión & $1-5$ & 2 & $2-10$ \\
\hline Corrección & $1-5$ & 3 & $3-15$ \\
\hline Adecuación & $1-5$ & 3 & $3-15$ \\
\hline Presentación & $1-5$ & 1 & $1-5$ \\
\hline Estilo & $1-5$ & 2 & $2-10$ \\
\hline PUNT. GLOBAL & & & $14-70$ \\
\hline
\end{tabular}

y (2) el Test de Habilidades Metalingüísticas 2 (THAM-2), de M. ${ }^{\mathrm{a}}$ A. Pinto, R. Titone y M. ${ }^{\mathrm{a}}$ D. González Gil (2000), instrumento de medida de habilidades lingüísticas (L) y metalingüísticas (ML) concebido para el tramo de edad comprendido entre 9 y 13 años, que consta de seis pruebas diferentes (comprensión, sinonimia, aceptabilidad, ambigüedad, función gramatical y segmentación fonémica), que se distribuyen en un total de 96 ítems, siendo las puntuaciones mínimas y máximas que pueden ser obtenidas en la valoración de habilidades metalingüísticas en esta prueba las que figuran en la siguiente tabla: 
Tabla 3. Puntuación de habilidades metalingüisticas del THAM-2.

\begin{tabular}{|l|c|}
\hline \multicolumn{1}{|c|}{ VARIABLES } & $\begin{array}{c}\text { HABILIDADES } \\
\text { METALINGÜÍSTICAS }\end{array}$ \\
\hline Comprensión & $0-32$ \\
\hline Sinonimia & $0-10$ \\
\hline Aceptabilidad & $0-26$ \\
\hline Ambigüedad & $0-14$ \\
\hline Función gramatical & $0-24$ \\
\hline Segmentación fonémica & $0-18$ \\
\hline PUNTUACIÓN GLOBAL & $0-124$ \\
\hline
\end{tabular}

\subsection{Proceso}

Las fases seguidas en este proceso han sido las siguientes: a) una fase inicial, durante la que se han realizado la revisión bibliográfica sobre investigaciones previas que pudieran aportar datos significativos en torno al tema, la concreción del marco teórico referido a enseñanza-aprendizaje de la composición escrita y al desarrollo de las habilidades metalingüísticas y la preparación de la investigación experimental. b) Una fase de desarrollo, que se ocupó en la elección del centro educativo para la realización de la investigación, la cumplimentación de los cuestionarios y realización de las correspondientes pruebas por parte de los alumnos(as) participantes en la investigación, la obtención de los resultados mediante el tratamiento estadístico apropiado de los datos y el análisis y discusión de los resultados obtenidos. Y c) una fase final, o de recopilación y sistematización del material teórico y empírico, y de elaboración y redacción, a modo de conclusión, de las oportunas consideraciones didácticas sobre habilidades metalingüísticas y desarrollo de capacidades para la composición de textos escritos.

\subsection{Estudios estadísticos realizados}

A fin de conseguir los objetivos planteados en esta investigación y de describir con la mayor precisión posible los fenómenos investigados, se han realizado los correspondientes análisis mediante la utilización del paquete de programas estadísticos SPSS en la versión 14.0 para Windows, a través de un proceso que ha constado de tres fases:

a) Una primera fase, en la que se ha llevado a cabo un análisis unidimensional de cada una de las variables estudiadas coincidentes con cada una de las distintas partes del THAM-2 así como de cada uno de los componentes de la Guía de evaluación de la composición escrita, con objeto de obtener de este modo los índices descriptivos más significativos de las mismas, tales como media, desviación típica, rango, mínimo, máximo, asimetría y curtosis.

b) Una segunda fase, en la que se ha realizado un primer análisis bivariable con el que se ha tratado de medir la magnitud y el signo de la relación lineal entre cada par de 
variables así como la comprobación de su significación estadística. Los índices obtenidos han sido el coeficiente de correlación $r$ de Pearson y la probabilidad del estadístico $t$ de Student.

c) Y una tercera fase, en la que se ha realizado un segundo análisis bivariable con el fin de estudiar la relación lineal entre cada una de las variables criterio (componentes de la composición escrita) y las variables predictoras (habilidades metalingüísticas), con objeto de predecir las puntuaciones de la variable criterio. En esta fase se han estudiado, asimismo, la fuerza global de la asociación $\left(R^{2}\right)$ y la significatividad de la relación (Anova), y se ha analizado la importancia de cada una de las variables predictoras (Coeficiente típico Beta).

\section{AnÁlisis de RESUltados obTenidos y CONSIDERACIONES DIDÁCtICAS}

\subsection{Estudios descriptivos: algunas reflexiones didácticas sobre las aportaciones de este estudio}

Los resultados obtenidos en los estudios descriptivos de las distintas variables son los que se reflejan a continuación:

Tabla 4. Estadísticos descriptivos de las variables de la investigación.

\begin{tabular}{lccccccc}
\hline \hline & Rango & Mínimo & Máximo & Media & $\begin{array}{c}\text { Desv. } \\
\text { Típ. }\end{array}$ & Asimetría & Curtosis \\
\hline Coherenc & 12,00 & 3,00 & 15,00 & 9,1023 & 2,8204 &,- 069 &, 001 \\
Cohesión & 12,00 & 3,00 & 15,00 & 9,3409 & 3,2302 &, 165 &,- 447 \\
Correcci & 12,00 & 3,00 & 15,00 & 9,5114 & 3,4707 &, 160 &,- 794 \\
Adecuaci & 12,00 & 3,00 & 15,00 & 9,6818 & 2,6021 &, 403 &, 139 \\
Presenta & 3,00 & 2,00 & 5,00 & 3,3409 &, 69293 &, 063 &,- 155 \\
Estilo & 8,00 & 2,00 & 10,00 & 5,2841 & 1,8442 &, 358 &,- 178 \\
TotalTE & 52,00 & 20,00 & 72,00 & 46,1818 & 12,063 &, 410 &,- 513 \\
ComprML & 27,00 & 3,00 & 30,00 & 13,3750 & 5,8098 &, 344 &,- 353 \\
SinonML & 6,00 &, 00 & 6,00 & 1,5682 & 1,5959 &, 880 &,- 038 \\
AceptML & 19,00 &, 00 & 19,00 & 11,1591 & 2,9976 &,- 463 & 1,375 \\
AmbigML & 12,00 &, 00 & 12,00 & 4,0568 & 2,640 &, 700 &, 151 \\
FungrML & 10,00 &, 00 & 10,00 & 3,1023 & 2,149 &, 703 &, 431 \\
SegfonML & 11,00 & 2,00 & 13,00 & 6,7386 & 2,3168 &, 519 &,- 033 \\
TotalML & 56,00 & 10,00 & 66,00 & 39,9886 & 11,06 &,- 038 &, 141
\end{tabular}


Los resultados que figuran en esta tabla dan pie a plantearse algunas reflexiones que pudieran ser de interés desde el punto de vista didáctico, pues, una vez conocidos los datos descriptivos de las variables referidas tanto a la composición escrita (coherencia, cohesión, corrección, adecuación, presentación, estilo y puntuación total de texto escrito) como a las habilidades metalingüísticas (comprensión, sinonimia, aceptabilidad, ambigüedad, función gramatical, segmentación fonémica y puntuación total de habilidades metalingüísticas), y expuestos para cada una de estas variables los estadísticos más significativos (rango, máximo y mínimo, media, desviación típica, asimetría, curtosis), se observa -a la vista tanto de los datos obtenidos en este estudio descriptivo como de los errores detectados en la corrección de las pruebas- que, a pesar de las múltiples diferencias puestas de manifiesto en muchos de los resultados, hay determinados aspectos de la composición escrita que podrían ser mejorados en el grupo de alumnos estudiado a través de la oportuna intervención didáctica.

\subsubsection{Reflexiones en torno a las variables referidas al texto escrito}

Teniendo en cuenta el estudio realizado sobre los resultados estadísticos de las variables investigadas, y a la vista de los índices más significativos que permiten un estudio comparativo de los mismos en todas las variables de la investigación, se puede constatar que respecto a la puntuación media obtenida por las diferentes variables valoradas en la prueba de composición escrita, se alcanza en todas ellas una media alta; no obstante, es necesario hacer algunas puntualizaciones sobre las distintas variables estudiadas.

En el caso de la coherencia, se habría de tener en cuenta -junto a los resultados obtenidos en el estudio descriptivo de esta variable- que en la corrección de las pruebas se ha observado que la mayor cantidad de fallos se acumula principalmente en la selección de la cantidad de información, en el orden de las ideas y en la correcta estructuración de los párrafos, y no tanto en el dominio de la lógica o de los principios generales del pensar ni en el conocimiento general de las cosas.

Teniendo en cuenta, pues, que los alumnos muestran un buen dominio referido a los principios generales del pensar y al conocimiento general de las cosas, la coherencia de sus escritos podría mejorar si la enseñanza se orientara más hacia el desarrollo de las capacidades para la generación de ideas y selección de las mismas (con objeto de que en el texto aparezca la información necesaria), hacia el dominio del orden que requiere la exposición de estas ideas y hacia el entrenamiento en la correcta estructuración del contenido a través de la debida disposición de los distintos párrafos del texto.

En cuanto a la cohesión textual y a la corrección idiomática conviene tener en cuenta que, si bien los resultados obtenidos en relación con la cohesión son muy parecidos a los de la variable coherencia, en la corrección de las pruebas se ha podido constatar que los fallos más abundantes se producen en el uso de enlaces y en la utilización de signos de puntuación. Asimismo, en el caso de la corrección idiomática, su similitud con los datos obtenidos en la variable cohesión -al ponerse de manifiesto una división importante de la muestra en ambas distribuciones entre alumnos con muy alto o muy bajo rendimiento- parece indicar la conveniencia de centrar las tareas docentes en la consecución de un mayor dominio ortográfico, sobre todo, mediante la realización de actividades específicas con el grupo de los alumnos que han mostrado que la mayoría de sus errores se manifiesta en el escaso dominio de la ortografía literal y de la acentuación, o, como ocurre en el caso de la variable cohesión, en 
el deficiente dominio en el empleo de los signos de puntuación, pues en ambos casos se trata de aspectos ortográficos, bien sean éstos referidos a la ortografía de la palabra o a la de la frase, y, tanto para un caso como para el otro, se reclama una instrucción específica y el necesario aprendizaje práctico.

A pesar de que en cuanto a la adecuación el rendimiento de los alumnos se muestra superior al que presentan en relación con el de otros componentes textuales, habría que seguir teniendo en cuenta que se trata de uno de los componentes de más difícil dominio para el alumnado de educación primaria, ya que tal dominio incluye el conocimiento de una serie de aspectos culturales, sociolingüísticos y pragmáticos que hacen difícil su aprendizaje y requieren tiempo y madurez personal para su plena adquisición.

El paralelismo que se existe en el rendimiento de los estudiantes entre presentación y estilo, según las distribuciones de la muestra reflejadas en sus correspondientes curvas de frecuencia, se ha venido poniendo de manifiesto en la corrección de las pruebas, a través de la cual se ha podido comprobar que los elementos propios del estilo -"elegancia en la expresión" y "creatividad expresiva"- suelen coincidir con las composiciones de mejor presentación.

\subsubsection{En cuanto a las variables correspondientes a las habilidades metalingüisticas}

Teniendo en cuenta los índices aparecidos en el estudio comparativo se puede constatar que en cuanto a las habilidades metalingüisticas, excepto para el caso de la variable segmentación fonémica, la puntuación media es muy baja para las variables comprensión metalingüistica, sinonimia metalingüistica, función gramatical metalingüística y segmentación fonémica metalingüística. Para la ambigüedad metalingüística, el histograma de frecuencias muestra una acumulación de los sujetos tendente hacia puntuaciones bajas por una parte y altas por otra, de manera claramente diferenciada por la patente disminución de frecuencias en la parte central; y solo en relación con la aceptabilidad metalingüística, la gráfica manifiesta una acumulación de los sujetos tendente hacia puntuaciones centrales.

A la vista de estos resultados, cabría entender que si se pretende mejorar la competencia de los alumnos para la elaboración de textos escritos, habría que hacer un esfuerzo importante para desarrollar sus habilidades metalingüísticas. Sería, pues, necesario utilizar estrategias que se centraran principalmente en ir elevando a nivel reflexivo (y siempre en la medida en que lo aconseje su desarrollo) lo que los escolares de primaria ya conocen y saben usar a nivel implícito, combinando el componente formal con el semántico con el fin de convertir así las estructuras de la lengua en repertorios de posibilidades para expresar ideas, sentimientos o vivencias mediante la expresión escrita.

\subsection{Estudio de correlaciones: consideraciones en torno a las correlaciones entre varia- bles}

En el terreno didáctico importa mucho conocer la posible tendencia a variar concomitantemente que pueden mostrar dos o más fenómenos presentes en la enseñanza; y, aunque no se pretenda con ello determinar la relación de causalidad existente entre fenómenos covariantes, sí es muy conveniente acercarse lo más posible al conocimiento del grado de enlace funcional que existe entre estos fenómenos, por cuanto pueden poner de manifiesto el 
influjo que unos componentes de la enseñanza tienen respecto a otros y orientar de esta manera la labor de maestros y profesores.

De aquí que, con objeto de medir la magnitud y el signo de la relación entre pares de variables cuantitativas de esta investigación, se haya procedido a realizar el estudio de su coeficiente de correlación, con la intención de poner de relieve los posibles factores influyentes en el rendimiento de los alumnos en composición escrita y poder juzgar con sólidos fundamentos sobre la importancia que presentan las distintas variables en relación con la adquisición de habilidades para componer textos escritos. Así pues, se han llevado a cabo los estudios de correlaciones entre las variables referidas tanto al texto escrito como a las habilidades metalingüísticas, independientemente consideradas y en su totalidad, en ambos casos, habiendo obtenido los resultados que se reflejan en la siguiente tabla: 


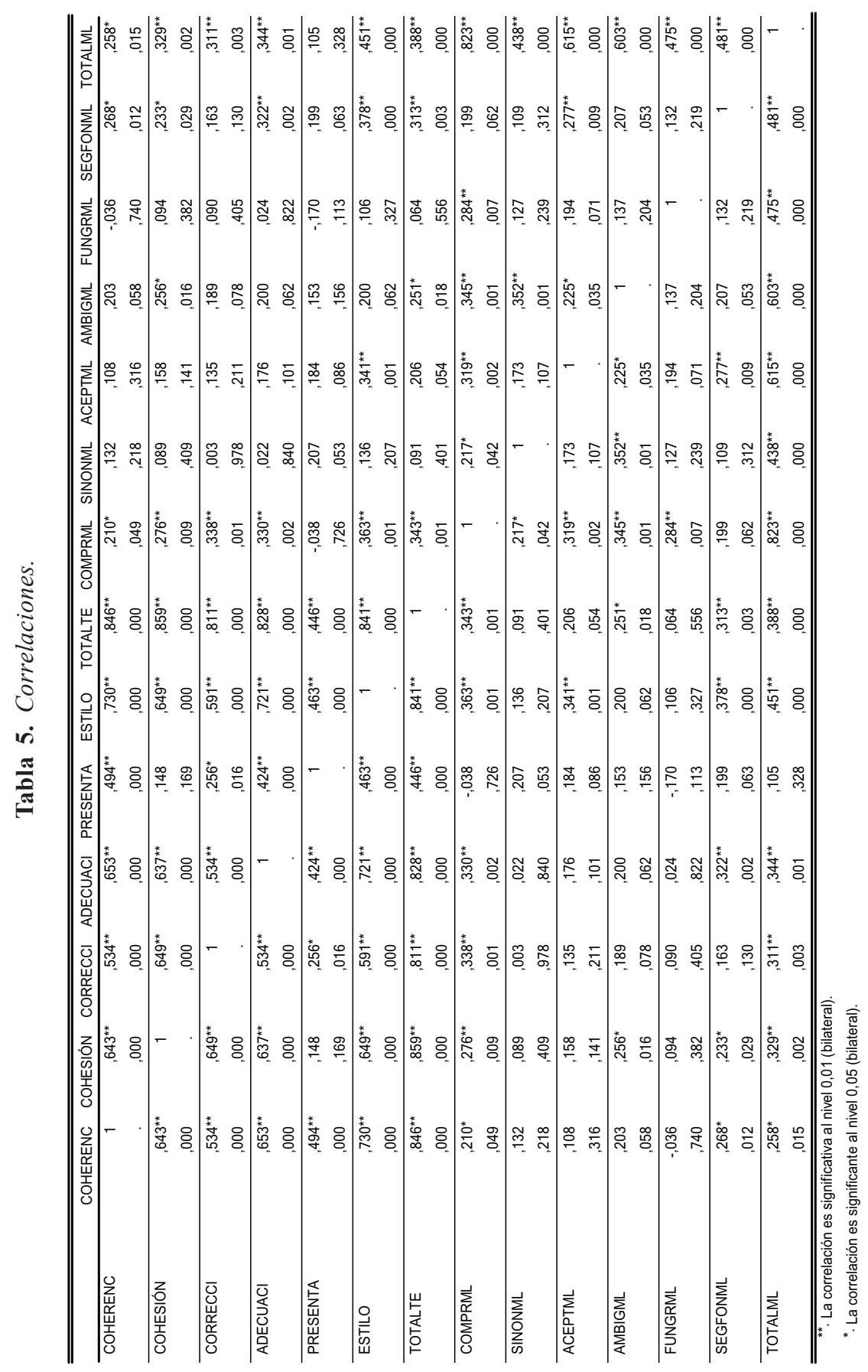


Los resultados obtenidos en el estudio de las correlaciones entre las variables referidas al texto escrito y a las habilidades metalingüísticas, muestran que la variable puntuación total de habilidades metalingüisticas y la variable comprensión metalingüistica son las únicas que correlacionan de manera significativa con seis de las siete que componen la prueba de composición escrita: estilo, puntuación total del texto escrito, adecuación, cohesión, corrección y coherencia. La variable segmentación fonémica metalingüística correlaciona a nivel significativo con cinco de las siete variables que componen la prueba de composición escrita: estilo, adecuación, puntuación total del texto escrito, coherencia y cohesión. La variable ambigüedad metalingüistica correlaciona a nivel significativo con dos de las siete variables que componen la prueba de composición escrita: cohesión y puntuación total del texto escrito. La variable aceptabilidad metalingüística correlaciona a nivel significativo sólo con una de las siete variables que componen la prueba de composición escrita: el estilo. Y las variables sinonimia metalingüistica y función gramatical metalingüistica no correlacionan de manera significativa con ninguna de las siete variables que componen la prueba de composición escrita.

Se hace patente, por tanto, que está claramente presente en los resultados obtenidos en este estudio la relación de reciprocidad entre el grado de dominio de habilidades metalingüísticas, globalmente consideradas, y el nivel de rendimiento en cada uno de los estándares de textualidad correspondientes a la composición escrita; pero, en este caso, también la habilidad comprensión metalingüistica, particularmente considerada, muestra esta misma tendencia a variar en la misma dirección acompañada de los componentes textuales de las composiciones escritas producidas por los escolares.

El hecho de que tanto el grado de desarrollo de la consciencia metalingüística, en general, como el de la comprensión metalingüística, en particular, muestren influencias recíprocas con el nivel de rendimiento en los distintos componentes textuales de la composición escrita, ha de hacer pensar en el efecto positivo que la labor docente puede tener cuando la intervención didáctica recae en el desarrollo de las habilidades metalingüísticas en su conjunto o en el de la comprensión metalingüística en concreto, puesto que cualquier mejora producida en las mismas tiende a verse acompañada por esta mejora en los distintos componentes textuales con los que correlacionan positivamente.

Por estas razones, también resulta de interés para la didáctica de la composición escrita conocer que, además del beneficio que aporta este desarrollo global de las habilidades metalingüísticas, entre estas habilidades, aquellas que presentan una mayor tendencia a variar y actuar conjuntamente en una misma dirección son la capacidad para captar el paso del plano del fonema al morfema (segmentación fonémica) y la capacidad para reestructurar el significado del texto en función de los cambios de significado producidos en lexemas (ambigüedad).

De igual manera, el hecho de que no existan correlaciones significativas entre las habilidades metalingüísticas sinonimia y función gramatical y el resto de componentes textuales, permite suponer que entre el desarrollo de estas habilidades metalingüísticas y el aprendizaje de la composición de textos escritos no existe una relación patente de covariación o reciprocidad directa, por lo que capacidades que tanto se cultivan en la escuela tales como las de determinar funciones gramaticales o analizar oraciones simples o compuestas, no parece que incidan con la misma importancia que demuestra tener el desarrollo de otras capacidades o habilidades metalingüísticas, entre las que ocupa el primer lugar la comprensión metalingüística, habilidad a la que, si bien por criterios estadísticos no pueda atribuírsele una relación causal, 
sí existen argumentos lógicos de solidez que permiten atribuir una importante y directa incidencia en todo proceso de elaboración textual.

\subsection{Consideraciones sobre los análisis de regresión}

Mediante el empleo en esta investigación de la técnica estadística de regresión múltiple, se ha pretendido estudiar la relación lineal entre las distintas variables criterio y las variables predictoras. En la realización de este análisis de regresión se han tomado como variables criterio cada una de las variables pertenecientes a la prueba de composición escrita, y como variables predictoras las variables correspondientes a las habilidades metalingüísticas. Los resultados obtenidos se exponen en la siguiente tabla:

Tabla 6. Análisis de regresión de las variables de la investigación.

\begin{tabular}{|c|c|c|c|c|c|c|c|}
\hline \multirow{2}{*}{$\begin{array}{l}\text { Variabl. } \\
\text { Criterio }\end{array}$} & \multirow{2}{*}{$\begin{array}{c}\text { Variables } \\
\text { Predictoras }\end{array}$} & \multirow{2}{*}{$\mathrm{R}^{2}$} & \multicolumn{2}{|c|}{ Anova } & \multicolumn{3}{|c|}{ Coeficientes } \\
\hline & & & $\mathrm{F}$ & Sign. & Beta & $\mathrm{t}$ & Sign. \\
\hline \multirow{7}{*}{$\begin{array}{l}\frac{\pi}{0} \\
\frac{\pi}{0} \\
\frac{0}{0} \\
\frac{0}{0}\end{array}$} & TotalML & \multirow{7}{*}{, 127} & \multirow{7}{*}{1,957} & \multirow{7}{*}{,081 } & 305 & 1,354 &, 180 \\
\hline & ComprML & & & & --- & --- & --- \\
\hline & SinonML & & & & ,012 & ,099 & ,922 \\
\hline & AceptML & & & &,- 097 &,- 693 &, 490 \\
\hline & AmbigML & & & &, 028 & ,202 &, 841 \\
\hline & FungrML & & & &,- 189 & $-1,500$ &, 137 \\
\hline & SegfonML & & & &, 166 & 1,357 &, 179 \\
\hline \multirow{7}{*}{ 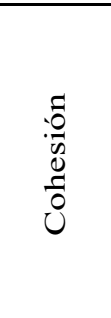 } & TotalML & \multirow{7}{*}{,132 } & \multirow{7}{*}{2,045} & \multirow{7}{*}{,069 } &, 356 & 1,587 &, 116 \\
\hline & ComprML & & & & -- & -- & --- \\
\hline & SinonML & & & &,- 082 &,- 693 &, 490 \\
\hline & AceptML & & & &,- 074 &,- 530 & ,598 \\
\hline & AmbigML & & & & ,080 & ,569 & ,571 \\
\hline & FungrML & & & &,- 072 &,- 574 &, 567 \\
\hline & SegfonML & & & & ,084 & ,690 &, 492 \\
\hline \multirow{7}{*}{$\begin{array}{l}\tilde{0} \\
0 \\
0 \\
0 \\
0 \\
0 \\
0\end{array}$} & TotalML & \multirow{7}{*}{, 138 } & \multirow{7}{*}{2,159} & \multirow{7}{*}{, 055} & .587 & 2,626 &, 010 \\
\hline & ComprML & & & & --- & --- & --- \\
\hline & SinonML & & & &,- 195 & $-1,650$ & ,103 \\
\hline & AceptML & & & &,- 151 & $-1,082$ &, 282 \\
\hline & AmbigML & & & &,- 039 &,- 279 &, 781 \\
\hline & FungrML & & & &,- 125 & $-1,000$ &, 320 \\
\hline & SegfonML & & & &,- 032 &,- 265 &, 792 \\
\hline
\end{tabular}


Tabla 6. Análisis de regresión de las variables de la investigación (Cont.).

\begin{tabular}{|c|c|c|c|c|c|c|c|}
\hline \multirow{2}{*}{$\begin{array}{l}\text { Variabl. } \\
\text { Criterio }\end{array}$} & \multirow{2}{*}{$\begin{array}{c}\text { Variables } \\
\text { Predictoras }\end{array}$} & \multirow{2}{*}{$\mathrm{R}^{2}$} & \multicolumn{2}{|c|}{ Anova } & \multicolumn{3}{|c|}{ Coeficientes } \\
\hline & & & $\mathrm{F}$ & Sign. & Beta & $\mathrm{t}$ & Sign. \\
\hline \multirow{7}{*}{ 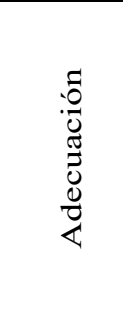 } & TotalML & \multirow{7}{*}{,196 } & \multirow{7}{*}{3,295} & \multirow{7}{*}{,006 } &, 545 & 2,523 &, 014 \\
\hline & ComprML & & & & -- & -- & -- \\
\hline & SinonML & & & &,- 171 & $-1,495$ &, 139 \\
\hline & AceptML & & & &,- 121 &,- 902 &, 370 \\
\hline & AmbigML & & & &,- 044 &,- 326 &, 745 \\
\hline & FungrML & & & &,- 203 & $-1,676$ & .098 \\
\hline & SegfonML & & & & ,148 & 1,256 & ,213 \\
\hline \multirow{7}{*}{ 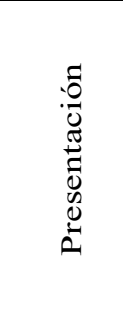 } & TotalML & \multirow{7}{*}{,160 } & \multirow{7}{*}{2,581} & \multirow{7}{*}{, 024} &,- 256 & $-1,158$ &, 250 \\
\hline & ComprML & & & & -- & -- & --- \\
\hline & SinonML & & & & ,222 & 1,904 & ,061 \\
\hline & AceptML & & & & 240 & 1,749 & ,084 \\
\hline & AmbigML & & & & ,152 & 1,104 &, 273 \\
\hline & FungrML & & & &,- 174 & $-1,407$ &, 163 \\
\hline & SegfonML & & & & ,223 & 1,857 & ,067 \\
\hline \multirow{7}{*}{ 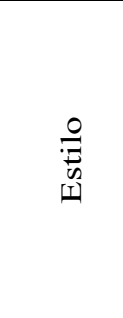 } & TotalML & \multirow{7}{*}{,260 } & \multirow{7}{*}{4,754} & \multirow{7}{*}{, 000} & ,471 & 2,322 &, 023 \\
\hline & ComprML & & & & -- & -- & --- \\
\hline & SinonML & & & &,- 050 &,- 455 &, 650 \\
\hline & AceptML & & & & ,055 & ,424 &, 672 \\
\hline & AmbigML & & & &,- 103 & -797 &, 428 \\
\hline & FungrML & & & &,- 136 & $-1,174$ & 244 \\
\hline & SegfonML & & & &, 176 & 1,564 & , 122 \\
\hline \multirow{7}{*}{ TotalTE } & TotalML & \multirow{7}{*}{,197 } & \multirow{7}{*}{3,318} & \multirow{7}{*}{,006 } & ,506 & 2,346 & ,021 \\
\hline & ComprML & & & & -- & -- & --- \\
\hline & SinonML & & & &,- 109 &,- 953 & ,344 \\
\hline & AceptML & & & &,- 090 &,- 671 & ,504 \\
\hline & AmbigML & & & & ,000 & ,000 & 1,000 \\
\hline & FungrML & & & &,- 162 & $-1,344$ & ,183 \\
\hline & SegfonML & & & & ,127 &, 085 & ,281 \\
\hline
\end{tabular}

Los resultados obtenidos muestran la aparición de una sola variable predictora significativa: la puntuación total de habilidades metalingüisticas. En los cuadros que se exponen a continuación se refleja esta variable y aquéllas a las que predice, indicando los coeficientes típicos Beta (primer cuadro) y el orden de importancia de cada una de las variables según su nivel de predicción (segundo cuadro). 


$\begin{array}{cccccccc} & \text { TotalTE } & \text { Coher } & \text { Cohes } & \text { Correc } & \text { Adecua } & \text { Presen } & \text { Estilo } \\ \text { TotalML } & , 506 & , 305 & , 356 & , 587 & , 545 & -, 256 & , 471\end{array}$

TotalML

$\begin{array}{ccccccc}\text { TotalTE } & \text { Coher } & \text { Cohes } & \text { Correc } & \text { Adecua } & \text { Presen } & \text { Estilo } \\ 3 & --- & --- & 1 & 2 & --- & 4\end{array}$

Ante estos resultados, es posible resaltar que:

a) La variable puntuación total de habilidades metalingüisticas es predictora significativa de cuatro variables referidas al texto escrito: corrección, adecuación, puntuación total de la prueba de texto escrito y estilo.

b) Las variables sinonimia, aceptación, ambigüedad, función gramatical y segmentación fonémica metalingüísticas no son predictoras significativas de ninguna variable de las contempladas en el texto escrito (coherencia, cohesión, corrección, adecuación, presentación, estilo y puntuación total de la prueba de texto escrito).

c) La variable comprensión metalingüística se introdujo también como posible variable predictora en el análisis de regresión múltiple, pero ha sido excluida en el modelo.

Así, pues, los resultados totales del Test de Habilidades Metalingüisticas (THAM) y los datos correspondientes a las habilidades metalingüísticas -pertenecientes a este test- muestran un alto grado de predicción sobre el nivel global de rendimiento en la producción de textos escritos; lo cual conduce a pensar en la gran influencia que ejerce el nivel de desarrollo de habilidades metalingüísticas sobre la calidad de las composiciones escritas producidas por los alumnos.

Por otra parte, si bien el nivel de desarrollo de las capacidades metalingüísticas incide significativamente y de manera global sobre la mayoría de los componentes del texto escrito, son el estilo y la adecuación (en este orden en que se mencionan) los dos componentes textuales que presentan un mayor índice de regresión y que, por tanto, reciben una mayor influencia de tales capacidades. Si bien con un menor índice de regresión, también la variable puntuación total de metalingüistica es predictora, aunque lo sea en menor grado, de la corrección textual y del texto escrito en general (TotalTE).

Tales resultados parecen bastante razonables si se tiene en cuenta que tanto el dominio de los distintos componentes del estilo (variedad y riqueza léxica, complejidad y variación sintáctica, elegancia en la expresión y creatividad expresiva) como el de los pertenecientes a la adecuación (discurso adecuado, apropiado y oportuno, claridad de propósitos y objetivos, y nivel de formalidad) o a la corrección (corrección morfosintáctica, corrección léxica y corrección ortográfica) y al conjunto del texto escrito (globalmente considerado) requieren disponer de la debida capacidad para reflexionar sobre el funcionamiento del lenguaje en sus niveles general, particular y textual.

En este proceso de desarrollo de habilidades metalingüísticas representan un importante papel los conocimientos gramaticales y el propio aprendizaje y uso de la lengua escrita escritura y lectura- que, en conexión con el resto de las actividades verbales -lengua oralde los aprendizajes culturales y de los sistemas de comunicación no verbal contribuirán decisivamente al desarrollo progresivo de la competencia comunicativa escrita. 


\section{A MOdo de SÍNTESIS Y DE CONCLUSIONES}

Las consideraciones didácticas derivadas de los resultados obtenidos en los estudios de correlaciones, habida cuenta las limitaciones propias de este trabajo, nos llevan a pensar que sería posible mejorar la enseñanza-aprendizaje de la composición escrita si se orientara el esfuerzo docente hacia el desarrollo de la consciencia metalingüística de los escolares, teniendo en cuenta que los trabajos más rentables podrían ser aquellos que tendieran al desarrollo de habilidades metalingüísticas en general y, sobre todo, de las relacionadas con: 1) la conciencia fonológica, puesto que los aprendices han de estar dotados de unos conocimientos sobre la estructura fonológica de las palabras que les permitan poder hacer explícitos sus conocimientos implícitos de manera cada vez más ágil y consciente; 2) la competencia semántica, que favorezca la elaboración de textos escritos y garantice la necesaria reestructuración de significados de sus composiciones escritas en relación con los cambios de significado producidos en los lexemas, distinguiendo en el contenido semántico de la palabra, como así lo entendieran Vygotsky (1934) y Coseriu (1992), entre referencia o designación, significado y sentido, por la esencial relación existente entre estos componentes semánticos y el hablar o el escribir de manera coherente, correcta y adecuada; y 3) la capacidad de comprensión metalingüistica, imprescindible para que los aprendices de la lengua escrita adquieran criterios claros que los capaciten para responder a las relaciones semántico-gramaticales y pragmáticas de cualquier tipo de composición y les propicien y activen formas superiores de pensamiento.

Asimismo, y pensando igualmente en proporcionar una mejora en la enseñanza-aprendizaje de la composición escrita, habría que tener en cuenta que los resultados del análisis de regresión confirman el alto grado de predicción de las distintas variables, consideradas en su totalidad, sobre el rendimiento de los escolares en la elaboración de textos escritos, y advierten sobre la necesidad de desarrollar sus habilidades metalingüísticas con objeto de que el conocimiento implícito sobre el lenguaje que en principio se presenta así en los niños, vaya haciéndose cada vez más explícito, a fin de que sus procesos de elaboración textual sean también cada vez más conscientes, reflexivos y críticos, ya que, como afirmara Luria. "el lenguaje escrito garantiza el control consciente sobre las operaciones que se realizan" (id.) y de que este paso del conocimiento implícito sobre la lengua al conocimiento reflexivo facilitará el desarrollo del aprendizaje de la escritura.

\section{REFERENCIAS BIBLIOGRÁFICAS}

Castelló Badía, M. (2000). "Las concepciones de los estudiantes sobre la escritura académica", en A. Camps y M. Milian (comps.). El papel de la actividad metalingüistica en el aprendizaje de la escritura. Santa Fe-Rosario (Argentina): Homo Sapiens, 67-104.

Coseriu, E. (1992). Competencia lingüística. Elementos de la teoría del hablar. Madrid: Gredos. Luria, A.R. (1979). Conciencia y lenguaje. Madrid: Visor, 1984.

Pinto, M. ${ }^{a}$ A.; Titone, R. y González Gil, M. ${ }^{a}$ D. (2000). La consciencia metalingüística. Teoría, desarrollo e instrumentos de medición. Pisa-Roma: Istituti Editoriali Poligrafici Internazionali.

Rijlaarsdam, G. y Couzijn, M. ([1996]2000). "La estimulación de la metacognición en la enseñanza de la escritura", en A. Camps y M. Milian (comps.): El papel de la actividad 
metalingüística en el aprendizaje de la escritura. Santa Fe-Rosario (Argentina): Homo Sapiens, 215-260.

Rodríguez Polo, J. y Romero López, A. (2004). Investigación en didáctica de la lengua y asesoramiento curricular. Un programa de intervención didáctica para la composición escrita de textos narrativos. Granada: Grupo Editorial Universitario.

Vygotsky, L. S. (1934). Pensamiento y lenguaje. Buenos Aires: La Pléyade, 1987. 